Relations industrielles

Industrial Relations

\title{
Le rationnel et le raisonnable: deux nécessités distinctes et conjugées en droit
}

\section{Fernand Morin}

Volume 41, numéro 3, 1986

URI : https://id.erudit.org/iderudit/050232ar

DOI : https://doi.org/10.7202/050232ar

Aller au sommaire du numéro

Éditeur(s)

Département des relations industrielles de l'Université Laval

ISSN

0034-379X (imprimé)

1703-8138 (numérique)

Découvrir la revue

Citer cet article

Morin, F. (1986). Le rationnel et le raisonnable: deux nécessités distinctes et conjugées en droit. Relations industrielles / Industrial Relations, 41(3), 578-582. https://doi.org/10.7202/050232ar
Résumé de l'article

Le rationnel et le raisonnable : deux necessites distinctes et conjugees en droit
Tous droits réservés (C) Département des relations industrielles de l'Universite Laval, 1986
Ce document est protégé par la loi sur le droit d'auteur. L'utilisation des services d'Érudit (y compris la reproduction) est assujettie à sa politique d'utilisation que vous pouvez consulter en ligne.

https://apropos.erudit.org/fr/usagers/politique-dutilisation/ 


\section{DISCUSSION}

\section{Le rationnel et le raisonnable}

\section{Deux nécessités distinctes et conjugées en droit}

\section{Fernand Morin}

En 1985, la Cour suprême du Canada retenait le critère du raisonnable pour justifier et, du même coup, limiter le contrôle judiciaire des sentences arbitrales ${ }^{1}$. Ces décisions firent grande impression si on en juge les rappels faits depuis en de nombreux jugements de la Cour supérieure et dé la Cour d'appel. Il peut être utile de souligner la justification de ce critère, de chercher à mieux connaître sa portée exacte et, si possible, de savoir comment on reconnaît ou distingue une décision raisonnable de celle que l'on qualifierait de déraisonnable. Si cette dernière opération était possible, alors on pourrait prévoir quand la Cour supérieure exercera utilement un contrôle.

Bien que le critère du raisonnable soit parfaitement acceptable (qui pourrait d'ailleurs ouvertement s'y opposer?), son application pratique peut être délicate et porter certaines difficultés en raison des entendements différents qu'il suggère et des renvois à des notions à contenu variable qu'il renferme. Pour cette raison d'ailleurs, nous avions déjà souligné qu'il pouvait être préférable de l'utiliser, pour fin de contrôle judiciaire, d'une manière négative à l'instar de messieurs les juges Beetz et Lamer qui employèrent 1'expression "déraisonnable» et même, «manifestement déraisonnable»" ${ }^{2}$ À notre avis, cette formulation négative comporte quelques avantages certains qu'il convient de rappeler:

- On laisse ainsi bien comprendre que ce contrôle judiciaire ne devrait s'effectuer que lorsqu'il se révèle absolument nécessaire au-delà des conceptions personnelles des agents de justice (Cour supérieure et Cour d'appel). N'est-ce pas d'ailleurs la portée de l'enseignement pratique de la Cour suprême du Canada! ${ }^{3}$

* MORIN, Fernand, professeur, département des Relations Industrielles, Université Laval, Québec.

** Syndicat des employés de production du Québec et de l'Acadie et CCRT et Société Radio-Canada (1984) 2RCS 412; Blanchard et Control Data Canada Ltd (1984) 2RCS 476.

1 F. MORIN, «Le raisonnable déraisonnable! ou la rationalité du raisonnable», $40 \mathrm{Rel}$. Ind., 646.

2 Monsieur le juge Beetz soulignait que le juge de contrôle n'aurait pas à qualifier la sentence arbitrale de bonne ou de mauvaise, mais qu'il doit se demander s'il s'agit d'une sentence arbitrale qui «peut se défendre». Notamment pour l'analyse des faits, monsieur le juge Lamer rappelait que l'arbitre était mieux placé que le juge de contrôle pour les apprécier (voir référence numéro 1).

3 C'était l'idée que nous voulions évoquer par le titre du commentaire: Le raisonnable déraisonnable! ou la rationalité du raisonnable, référence numéro 2. 
- Ce qui peut ne pas nous paraître personnellement raisonnable peut cependant ne pas être véritablement déraisonnable. Parfois, cette nuance peut être déterminante pour exercer ou refuser ce contrôle judiciaire. Cette distinction fait moins appel à la subjectivité du juge de contrôle et plus, à l'entendement général du raisonnable, du tolérable, c'est-à-dire de ce qui ne choque pas, parce que incongru, injuste ou franchement incohérent en fonction de l'ensemble de règles de droit applicables.

- Cette approche limitative et circonscrite respecte l'économie du régime puisqu'il s'agit de poser un acte lui-même négatif et contraire au cours normal et régulier du système de justice retenu par le législateur en matière de griefs (articles 101, 106 et 107, Code du travail).

- Il s'agit d'une notion juridique que le droit public nord-américain et anglais utilise depuis fort longtemps et comprend un acte qu'aucune personne de bon sens n'aurait posé, en semblables circonstances.

Quelle que soit la formulation retenue, le raisonnable ou le non-déraisonnable, ce critère fait néanmoins appel à la raison et ainsi conforte l'esprit de justice et d'équité ou, selon le cas, rend compte d'un système juridique que l'on veut stable, complet et cohérent, du moins à titre d'objectif ou de postulat. Pour nos propos, nous ne pouvons qu'être d'accord pour que ce recours judiciaire puisse permettre d'écarter les scories, les entorses au règne de la raison, de la cohérence et du juste. Il nous faut reconnaître que ce critère ne répond pas d'une logique pure et qu'il véhicule une certaine charge de subjectivité. Pour ces raisons, il ne peut qu'imposer ses propres limites si on reconnaît sa rationalité, sa logique interne et sa dynamique ${ }^{4}$. C'est d'ailleurs cette même idée que rappelait ainsi Lord Hailsham: «Two reasonable persons can perfectly reasonably come to opposite conclusions on the same facts without forfeiting their title to be regarded as reasonable $\aleph^{5}$. On peut croire aussi que la Cour suprême du Canada devra expliciter davantage cette notion du raisonnable puisqu'elle sert de critère principal pour établir la constitutionalité des lois en fonction de la Charte canadienne des droits et libertés (art. 1). Entre-temps, cette question intéresse toujours les juristes dont le professeur Claude D'Aoust. Ce dernier voulut apporter, dit-il, quelques précisions sur le rationnel et le raisonnable et nous offrir, par le biais des précisions apportées, quelques moyens pour éviter ce contrôle judiciaire ${ }^{6}$. Cette dernière contribution, un peu comme une course à relais, nous incite à poursuivre l'analyse de la portée réelle ou virtuelle du raisonnable à titre de critère de contrôle judiciaire des sentences arbitrales.

Il s'agit certes d'un lieu commun que d'affirmer que le rationnel se distingue du raisonnable, bien que le premier puisse servir de voie utile pour conduire au dernier. Malgré leur parenté éthymologique, il demeure néces-

4 In Re W., 1971, A.C., 682 a 700.

5 Claude D'AOUST, Le contrôle judiciaire des décisions arbitrales, Réflexions sur les moyens d'y échapper; 41 Rel. Ind., 348.

6 S'il en est généralement ainsi, l'histoire nous fournit bien des exemples contraires où on oeuvra rationellement à la réalisation d'objectifs diaboliques. 
saire, en droit comme en bien d'autres disciplines, de ne pas confondre ces deux notions pour mieux savoir les utiliser et même, les conjuguer. Ainsi, on qualifiera généralement de rationnel un moyen, une approche, un modèle, une façon de faire, de penser, alors que l'autre, le raisonnable, réfère ou renvoie davantage au résultat, à l'aboutissement, à la conclusion anticipée ou finale. Selon cette division, la rationalité s'entend de l'adaptation au mieux des moyens aux fins, c'est-à-dire du choix des moyens les plus appropriés, les plus intelligents, logiques ou efficaces en fonction d'objectifs prédéterminés. Ainsi compris, le processus rationnel ne comporte cependant pas une garantie directe, nécessaire et certaine que la fin recherchée, le résultat obtenu ou la conclusion terminale soit raisonnable; cependant, cette fin devrait aussi être rationnelle. Bien souvent, ce ne sont ni le moyen ni la fin qui sont rationnels mais bien l'intime rapport les réunissant. Règle générale, il est vrai, l'homme capable de choisir de tels moyens logiques, cohérents et appropriés aura sans doute anticipé un objectif qui ne choque pas la raison et qui soit lui-même logique et cohérent si on le situe dans un plus grand ensemble ${ }^{7}$. Sous cette approche relative, il faut reconnaître que cette première fin ou conclusion peut aussi constituer un moyen simple, une première étape d'une fin plus générale et ainsi de suite. Ainsi, ne pourrait-on d'abord aboutir par voie rationnelle (analyse logique, syllogisme, etc.) qu'à une décision arbitrale rationnelle compte tenu de l'ensemble du droit applicable (l'économie générale du Code du travail et de la convention collective). Par ailleurs, cette même décision arbitrale rationnelle ne saurait être, de ce seul fait, raisonnable.

Le raisonnable s'entend d'abord ou généralement de ce qui se dégage ou résulte, de ce qui est conforme ou de ce que permet la saine raison dans ce que l'on ressent communément comme étant juste, légitime, conforme à l'entendement commun des choses. Si d'expérience il en est ainsi, on sait également qu'une décision ne provenant pas directement d'un processus rationnel pourrait aussi être qualifiée de raisonnable selon diverses autres acceptions:

1) parce que son dispositif ou sa conclusion satisfait ou répond à l'entendement commun de justice en un milieu donné;

2) parce qu'elle est construite de manière à respecter ou du moins, à ne pas heurter les règles principales du droit applicable ou l'économie générale du système juridique ou encore, parce qu'on la considère bien adaptée à la situation de fait et de droit;

3) parce que la démarche, l'analyse et les motifs invoqués pour y aboutir sont suffisamment convaincants et rassurants pour que la conclusion retenue ou qui s'en dégage soit bien acceptée et jugée elle-même satisfaisante et partagée à ce titre par la grande majorité des interlocuteurs.

On comprend alors qu'il serait possible qu'un juge de contrôle puisse déclarer raisonnable ou non déraisonnable la même sentence arbitrale en s'autorisant de l'un de ces trois entendements, mais pas nécessairement des

7 La même sentence arbitrale visée dans l'affaire Control Data (référence 1) n'a-t-elle pas connu cette double qualification selon les instances en présence? 
trois à la fois. Selon ces trois mêmes acceptions, ne peut-on pas croire qu'un juge puisse procéder à rebours et conclure, sous l'un ou l'autre de ces mêmes chefs, que la sentence arbitrale lui paraît déraisonnable ${ }^{8}$ ? Ce qui indique, à tout le moins, qu'il ne s'agit nullement d'un critère sûr et objectif. S'il n'y a pas lieu en droit d'en faire un quelconque ersatz, il faut cependant reconnaître la qualité véritable de ce critère notamment celle d'imposer une recherche respectueuse du droit à l'occasion de l'exercice de ce contrôle. Pourquoi devrait-il en être ainsi?

Si la démarche rationnelle et la conclusion raisonnable peuvent être si différentes et non liées nécessairement en un rapport causal, pourquoi cherche-t-on l'emprunt de voies rationnelles et logiques pour atteindre une conclusion qualifiée, en droit, de raisonnable ou, à tout le moins, de nondéraisonnable? D'une certaine manière, nous pourrions répondre que l'on agit ainsi pour que le droit nous soit plus tolérable en laissant moins de place, dans l'aménagement des rapports sociaux, à l'arbitraire, aux passions, à la vindicte. On utilise, de cette façon, un moyen que l'on peut partager avec tous les intéressés, selon une même lecture culturelle et historique, et qui permet de convaincre plus par l'esprit et en faisant surtout appel à l'intelligence. À ces fins, nous cherchons à ce que les règles de droit soient logiques, construites et aménagées sur des bases rationnelles et qu'elles soient d'une application raisonnable. À titre de simples rappels de cette construction, pensons aux multiples règles d'interprétation des règles de droit qui reposent sur des postulats qui présument ou font appel à l'intelligence, à la logique et à la raison.

- le législateur ne parle pas pour ne rien dire: présomption d'intelligence de l'objet traité et de la manière d'en traiter;

- les voies et moyens retenus en une loi sont appropriés à sa fin et à nulle autre: présomption d'un choix logique et pertinent pour établir cette adéquation;

- chaque partie d'une loi s'entend et se comprend à l'aide de l'ensemble et de manière à donner au tout cohérence et harmonie: postulat de plénitude du droit de manière à éviter l'arbitraire et à sécuriser;

- les règles de droit se situent entre elles selon certains ordres hiérarchiques: celui des sources (constitution, lois, règlements, contrats, etc.); celui du temps (la règle postérieure supplante la règle antérieure); celui de la spécificité (la règle particulière exclut, dans cette seule mesure, la règle générale), etc.: postulats d'une construction intelligente, logique et harmonieuse du droit de manière à obtenir sécurité et stabilité et à respecter la séparation des pouvoirs.

N'utilise-t-on pas aussi des normes d'évaluation du comportement des justiciables et qui reposent sur le rationnel et le raisonnable? Le modèle du «bon père de famille» c'est-à-dire de la personne prévoyante, prudente et raisonnable sert également à objectiver le droit, à le rendre au-dessus de

8 Une récente décision de la Cour suprême où on reconnut la compétence exclusive de l'arbitre de grief pour imposer réparation à la suite d'une violation de la convention collective repose sur ce postulat: St. Anne MacGamic Pulp and Paper et Syndicat canadien des travailleurs du papier (no. 17485) du 12 juin 1986. 
chaque individu et ainsi, plus acceptable à chacun puisque, par ce modèle transcendant, on aime croire que le juge juge les autres non selon lui, mais en les comparant à la conduite présumée du «bon père». Pour les fins de l'administration de la justice et l'application in concreto du droit, autant plaideurs que juges et arbitres emploient la méthode discursive pour convaincre ou vaincre. Ils tentent respectivement de faire appel à la raison pour convaincre que justice serait ainsi rendue et que la conclusion proposée ou qui découle de leur cheminement rationnel serait inévitablement la bonne ou relativement la meilleure et à défaut, qu'elle serait acceptable, supportable, voire légitime. Lorsque l'application d'une règle paraît pénible en un cas particulier, le juge ne démontre-t-il pas qu'il est préférable de l'imposer au lieu de retenir une avenue plus discrétionnaire? N'est-ce pas l'idée sousjacente invoquée par le brocard «Dura lex sed lex»?

En somme, nous voulons une justice qui soit administrée avec cohérence, logique et régularité pour éviter les surprises de l'arbitraire, de l'improvisation et du caprice. Â ces fins, on recherche une démonstration pour convaincre que justice est bien rendue et que la conclusion est logique, cohérente et régulière compte tenu du cadre juridique dans lequel elle s'insère et aussi, s'infère. Quoiqu'il en soit, une décision de justice comporte toujours un certain jugement de valeur et, par cette démonstration, on souhaite pouvoir la partager, la faire accepter.

S'il en est ainsi en droit, règle générale, il nous faut reconnaître la justesse de ce tandem raisonnable-déraisonnable en ce domaine de l'arbitrage des griefs:

- raisonnable la sentence arbitrale! Ce qui comprend, à notre point de vue, l'obligation de l'arbitre de démontrer logiquement et rationnellement la qualité de sa conclusion de manière à convaincre d'abord les justiciables et éventuellement le juge qu'elle est raisonnable c'est-à-dire respectueuse du droit et des faits;

- déraisonnable la sentence arbitrale! Elle doit seule alors être écartée par le juge de contrôle de manière à éviter l'arbitraire, le caprice, l'abus de pouvoir. Il revient alors au juge de démontrer cet aspect déraisonnable de la sentence et non seulement de le proclamer. Il doit en être ainsi si l'on veut respecter la répartition des fonctions et l'économie générale du droit du travail ${ }^{9}$.

En somme, ces deux facettes de ce janus comprennent des obligations sérieuses et pour l'arbitre et pour le juge et aussi, un mutuel respect de leur mission respective. N'est-ce pas des conditions nécessaires pour atteindre trois objectifs du droit: justice, sécurité et liberté? 